\title{
The potential of signal transduction inhibitors for the treatment of arthritis: is it all just JNK?
}

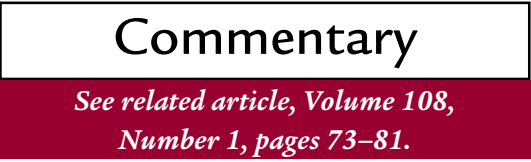

\author{
Matthew P. Vincenti ${ }^{1}$ and Constance E. Brinckerhoff 1,2 \\ ${ }^{1}$ Department of Medicine, and \\ ${ }^{2}$ Department of Biochemistry, Dartmouth Medical School, Hanover, New Hampshire, USA
}

Address correspondence to: Constance E. Brinckerhoff, Department of Medicine, Dartmouth Medical School, Hanover, New Hampshire 03755, USA. Phone: (603) 650-1609; Fax: (603) 650-1128; E-mail: constance.e.brinckerhoff@dartmouth.edu.

J. Clin. Invest. 108:181-183 (2001). DOI:10.1172/JCI200113508.

Rheumatoid arthritis (RA) is a connective tissue disease that affects more than $1,000,000$ people in the US (1). This autoimmune disorder is driven largely by the recruitment of activated immune cells ( $T$ and B cells) and macrophages to the afflicted joints. There, the cytokines IL-1 and TNF, which are produced by these cells, mediate the irreversible joint destruction seen in RA (2). The downstream genes activated by these cytokines encode both inflammatory molecules and secreted proteinases of the matrix metalloproteinase (MMP) family (3).

The interstitial collagenases (collagenase-1, MMP-1; collagenase-2, MMP-8; collagenase-3, MMP-13; MT1-MMP, MMP-14) act at a committed step in the progression of RA, degrading type II collagen in cartilage $(1,3)$. Of these enzymes, particular attention has focused on MMP-1 and MMP-13 because they are induced in response to IL-1 and TNF and are found at elevated levels in RA (4). Therefore, inhibition of cytokine-induced MMP-1 and MMP-13 gene expression has been a prime target for the development of new arthritis drugs.

Earlier studies identified sequences in the promoters of these two MMP genes that mediate the increase in gene expression, including a proximal AP-1 site that binds members of the Fos and Jun families of transcription factors (5, $6)$, and several upstream sequences such as an NF-KB-like element (7) and a Cbfa1/OSF2/RUNX-2 (RUNX-2) binding site (8). Mutational and deletional analysis has indicated that these upstream sites cooperate with the proximal AP-1 site in order to drive transcription $(7,9,10)$. Further, although both MMP-1 and MMP-13 expression increases with cytokine stimulation, this increase is somewhat cell type-restricted and differs somewhat between the two genes. The restricted expression of MMP-13 in osteoblasts and chondrocytes may reflect the predominant expression in these cells of RUNX-2, which activates the MMP13 but not the $M M P 1$ promoter $(8,11)$ (J.A. Mengshol and C.E. Brinckerhoff, unpublished work). In contrast, cytokines induce MMP-1 expression in virtually all human connective tissue cells (4), clearly indicating that the mechanisms controlling expression of these two collagenases differ.

Recent studies have begun to analyze signal transduction pathways for their possible role in the transcriptional activation of MMP-1 and MMP-13 in cells associated with arthritic disease. The enzymes that

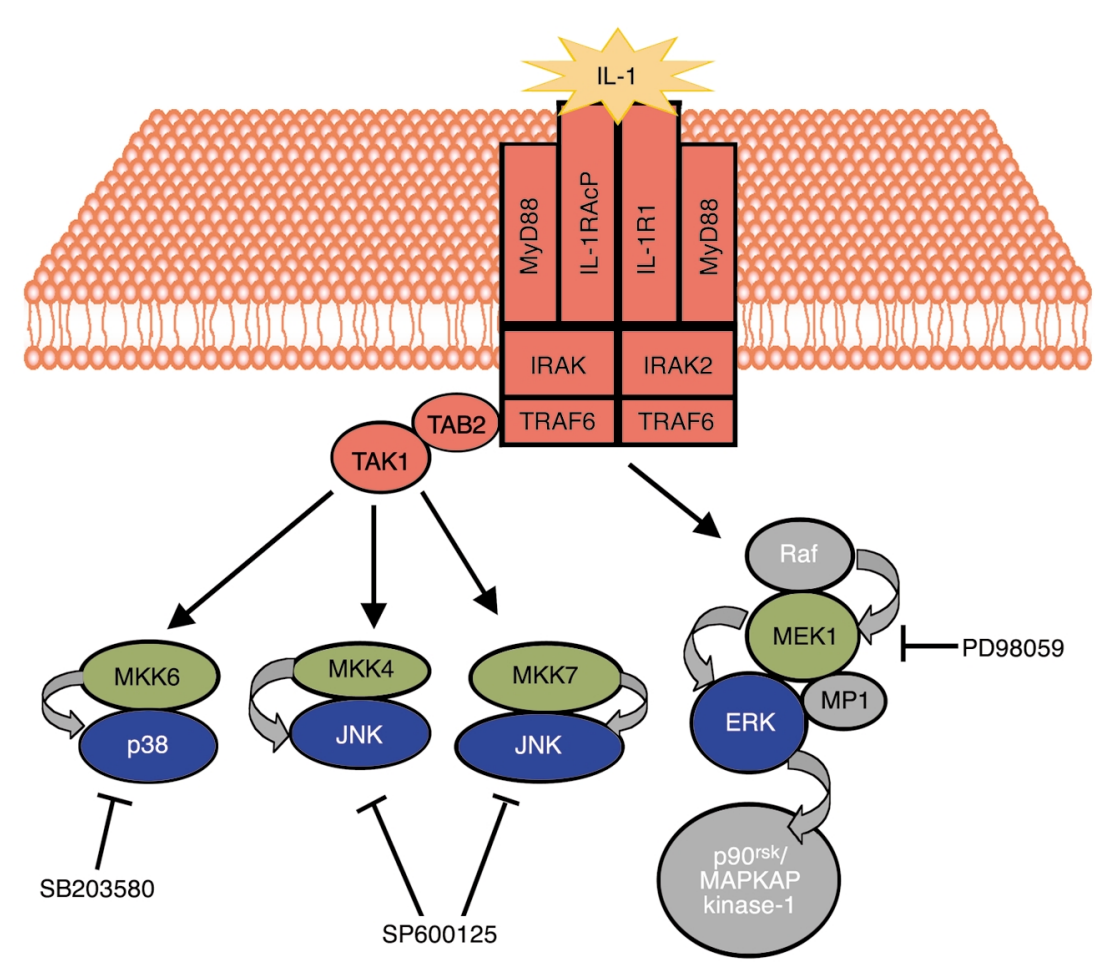

Figure 1

IL-1 activation of MAPK pathways. IL-1 binds to its cellular receptor and activates receptor-associated proteins (red), such as IRAK, TRAF6, and TAK1. The result is activation of several MAPK kinases (MKK6, MKK4, MKK7, and MEK1; green), which phosphorylate and active the MAPKs p38, JNK, and ERK (blue). SB203580 inhibits the p38 kinases, while PD98059 blocks activation of MEK1, and as a consequence ERK activation. SP600125 is a novel inhibitor of JNK, described by $\mathrm{Han}$ et al. in a recent issue of the $J C l(13)$. 


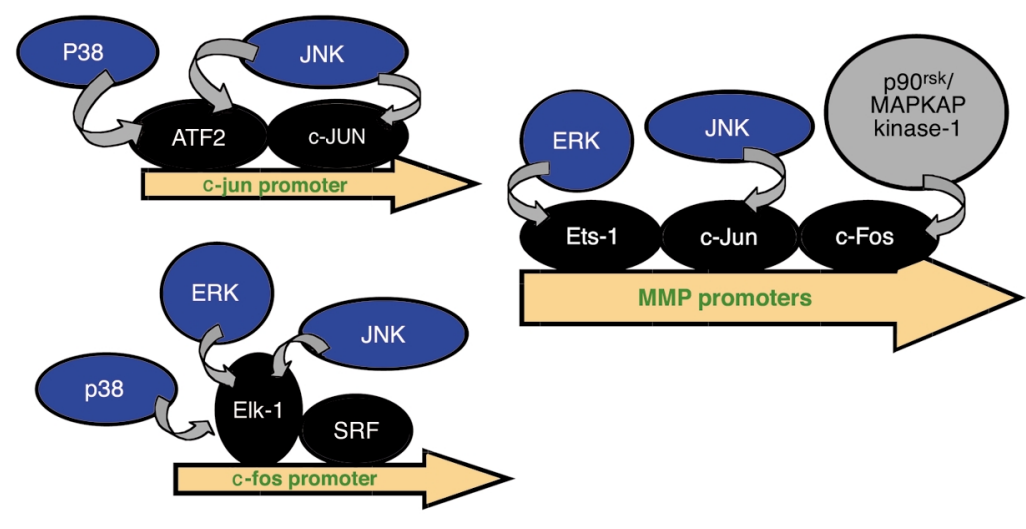

Figure 2

MAPK-inducible genes involved in inflammation and connective tissue breakdown. Activated MAPKs (blue) translocate to the nucleus, where they phosphorylate specific transcription factors (black), such as ATF2, c-Jun, Ets-1, c-Fos, and Elk-1. Activation of these transcription factors results in increased transcription from promoters of genes involved in inflammation and connective tissue disease, including those for c-jun, c-fos, and the MMPs.

facilitate these pathways are termed the mitogen-activated protein kinases (MAPKs) (12) and include the c-Jun $\mathrm{N}$-terminal kinases (JNKs), the extracellular signal-regulated kinases (ERKs), and the p38 kinases. The availability of chemical compounds that can specifically block one or another signal transduction pathway has greatly aided these studies (Figure 1). In a recent issue of the JCI, Han et al. (13) in the laboratory of Gary Firestein describe the ability of a new inhibitor, SP600125, to specifically block JNK, thus providing a tool to investigate the role of this kinase in MMP gene expression and in the pathophysiology of RA. They show that SP600125 completely blocks IL-1-induced expression of c-Jun and collagenase mRNAs and prevents the accumulation of phospho-Jun in cultured synovial cells. The in vitro binding of nuclear proteins to an AP-1-containing DNA fragment is likewise severely inhibited, consistent with evidence that this cis-acting sequence is a major target of IL-1 induction, apparently as a result of JNK activation.

These results may suggest a central role for the pathway, at least in cultured cells, but the data are slightly less clear-cut in the animal studies, since the authors observe incomplete silencing of collagenase mRNA induction and only a partial inhibition of joint destruction in SP600125-treated rats. Other pathways may contribute to this pathological process. Alternatively, other cells, in addition to syn- ovial fibroblasts, or other proteinases, in addition to the well studied MMPs, may be involved joint destruction.

\section{Functions for JNK and the other MAPKs}

Initially, it was believed that the JNK and $\mathrm{p} 38$ pathways alone were responsible for mediating responses to inflammatory cytokines (Figure 1), agents that induce apoptosis, and osmotic shock (14). These kinases act, in part, by phosphorylating and activating the transcription factors c-Jun and ATF2 (Figure 2). Upon activation, these transcription factors can induce the promoters of multiple genes, including those for MMPs and for c-Jun itself. In contrast, the ERKs were thought to be responsible for transducing growth factor-dependent proliferative signals, because they phosphorylate and activate Elk-1, which transactivates serum response elements (15). Unfortunately, the roles of individual MAPKs are not this discrete. Indeed, IL-1 is a potent activator of all three MAPK pathways (10) (Figure 1), and all MAPKs can activate the transcription factor Elk-1, which mediates growth factor responses by activating the c-fos promoter (16, 17) (Figure 2).

Chemical inhibition of the ERK pathway with PD98059 has raised additional issues, as Han et al. showed in previous work (19). PD98059 can specifically inhibit MEK1 and MEK2, two enzymes that phosphorylate and activate ERK1 and ERK2. When cells are treated with this compound and stimulated with serum, phorbol esters, or growth factors, proliferative responses are blocked, as expected. However, when this compound is added to IL-1-stimulated synovial fibroblasts, activation of MMP-1 is also significantly reduced (10). Indeed, Han et al. also found that PD98059 reduces both c-Jun phosphorylation and MMP-1 gene expression in IL-1-stimulated fibroblast-like synovial cells (13). Although this inhibition appears less effective than that conferred by the JNK inhibitor, such evidence raises doubts about the existence of MAPK pathways that are specific for either inflammation or degradation.

MAPK specificity may exist with respect to MMP-1 and MMP-13 gene regulation. Although all three MAPK pathways can contribute to MMP-1 expression in synovial fibroblasts (10, 19), cytokine activation of MMP-13 in chondrocytic cells appears to be primarily $\mathrm{p} 38$-dependent, since it is blocked with the p38 kinase inhibitor SB203580 (Figure 1) (20). While the specific nuclear targets of $\mathrm{p} 38$ in chondrocytic cells have not been precisely defined, they may include the chondrocyte- and osteoblast-specific transcription factor RUNX-2. In contrast, Han et al. found that the $\mathrm{p} 38$ pathway plays no significant role in MMP-13 expression in rat synovial-like fibroblasts (13). This apparent contradiction may reflect inherent differences between the human and the rat and mouse MMP13 genes: Rodents do not possess a homologue of the human $M M P 1$ gene (21). In these species, MMP-13 must function as the cytokine-inducible collagenase in both fibroblasts and chondrocytes; this is unlike the situation in humans, where MMP-13 expression is restricted to chondrocytes and osteoblasts and MMP-1 predominates in cytokinetreated fibroblasts. Therefore, the regulation of MMP-13 in rats and mice may be slightly different from that in humans, resulting in a greater dependence on the JNK pathway.

Han et al. (13) have presented a detailed analysis of a JNK-specific inhibitor that appears to be quite effective at inhibiting AP-1 function and collagenase gene expression, both in vitro and in vivo. However, it is important to point out that in vivo, the chondroprotection they observed was not complete, again emphasizing the complexity of mechanisms regulating 
MMP gene expression. One of the most interesting and potentially clinically relevant findings is that this inhibitor blocks collagenase gene expression and connective tissue destruction in vivo, while having little effect on inflammation (as assessed by foot pad swelling in mice). This result is consistent with the long-known limitation of nonsteroidal anti-inflammatory drugs (NSAIDS), which inhibit inflammation without impeding cartilage degradation (1). Thus, while inflammation is an important initiator of irreversible joint destruction, the specific intracellular pathways involved appear to be distinct. This study is a major contribution to understanding RA pathophysiology, because it provides a new tool for assessing the role of the JNK pathway in MMP gene expression in multiple cell types. For instance, it will be important to establish whether inflammatory cytokines can induce MMP-13 expression in JNK-inhibited chondrocytes. However, given the established roles of MMP-1 and MMP-13 in human RA, and the cell type-specific regulation of these genes, it is likely that the JNK, ERK, and p38 pathways will have to be targeted in concert if MAPK-inhibitory compounds are to be clinically effective in the treatment of RA.

\section{Acknowledgments}

The authors would like to acknowledge J.A. Mengshol for critical reading of this manuscript, and financial sup- port from the National Institute of Arthritis and Musculoskeletal and Skin Diseases (AR-46977 and AR02024 to M.P. Vincenti; AR-26599 to C.E. Brinckerhoff), the National Cancer Institute (CA-77267 to C.E. Brinckerhoff), and the Ronya and George Kozmetsky Foundation (Austin, Texas, USA; to C.E. Brinckerhoff) for funding of this research.

1. Ruddy, S., Harris, E.D., Sledge, C.B., and Kelley, W.N. 2001. Kelley's textbook of rheumatology. W.B. Saunders Co. Philadelphia, Pennsylvania, USA. 1904 pp.

2. Arend, W.P., and Dayer, J.M. 1995. Inhibition of the production and effects of interleukin-1 and tumor necrosis factor alpha in rheumatoid arthritis. Arthritis Rheum. 38:151-160.

3. Nagase, H., and Woessner, J.F., Jr. 1999. Matrix metalloproteinases. J. Biol. Chem. 274:21491-21494.

4. Vincenti, M.P. 2001. The matrix metalloproteinase (MMP) and tissue inhibitor of metalloproteinase (TIMP) genes. Transcriptional and posttranscriptional regulation, signal transduction and cell-type-specific expression. Methods Mol. Biol. 151:121-148.

5. Auble, D.T., and Brinckerhoff, C.E. 1991. The AP1 sequence is necessary but not sufficient for phorbol induction of collagenase in fibroblasts. Biochemistry. 30:4629-4635.

6. Angel, P., et al. 1987. Phorbol ester-inducible genes contain a common cis element recognized by a TPA-modulated trans-acting factor. Cell. 49:729-739.

7. Vincenti, M.P., Coon, C.I., and Brinckerhoff, C.E 1998. Nuclear factor kappaB/p50 activates an element in the distal matrix metalloproteinase 1 promoter in interleukin-1beta-stimulated synovial fibroblasts. Arthritis Rheum. 41:1987-1994.

8. Jimenez, M.J., et al. 1999. Collagenase 3 is a target of Cbfa1, a transcription factor of the runt gene family involved in bone formation. Mol. Cell. Biol. 19:4431-4442.

9. Porte, D., et al. 1999. Both AP-1 and Cbfa1-like factors are required for the induction of interstitial collagenase by parathyroid hormone. Oncogene. 18:667-678.
10. Barchowsky, A., Frleta, D., and Vincenti, M.P. 2000. Integration of the NF-kappaB and mitogenactivated protein kinase/AP-1 pathways at the collagenase-1 promoter: divergence of IL-1 and TNFdependent signal transduction in rabbit primary synovial fibroblasts. Cytokine. 12:1469-1479.

11. Karsenty, G. 1998. Transcriptional regulation of osteoblast differentiation during development. Front Biosci. 3:D834-D837.

12. Garrington, T.P., and Johnson, G.L. 1999. Organization and regulation of mitogen-activated protein kinase signaling pathways. Curr. Opin. Cell Biol. 11:211-218.

13. Han, Z., et al. 2001. c-Jun N-terminal kinase is required for metalloproteinase expression and joint destruction in inflammatory arthritis. $J$. Clin. Invest. 108:73-81.

14. Karin, M. 1995. The regulation of AP-1 activity by mitogen-activated protein kinases. Cell. 270:16483-16486.

15. Gille, H., et al. 1995. ERK phosphorylation potentiates Elk-1-mediated ternary complex formation and transactivation. EMBOJ. 14:951-962.

16. Whitmarsh, A.J., Yang, S.H., Su, M.S., Sharrocks, A.D., and Davis, R.J. 1997. Role of p38 and JNK mitogen-activated protein kinases in the activation of ternary complex factors. Mol. Cell. Biol. 17:2360-2371.

17. Whitmarsh, A.J., Shore, P., Sharrocks, A.D., and Davis, R.J. 1995. Integration of MAP kinase signal transduction pathways at the serum response element. Science. 269:403-407.

18. Alessi, D.R., Cuenda, A., Cohen, P., Dudley, D.T., and Saltiel, A.R. 1995. PD 098059 is a specific inhibitor of the activation of mitogen-activated protein kinase kinase in vitro and in vivo. J. Biol. Chem. 270:27489-27494.

19. Han, Z., et al. 1999. Jun N-terminal kinase in rheumatoid arthritis. J. Pharmacol. Exp. Ther 291:124-130.

20. Mengshol, J.A., Vincenti, M.P., Coon, C.I., Barchowsky, A., and Brinckerhoff, C.E. 2000. Interleukin-1 induction of collagenase 3 (matrix metalloproteinase 13) gene expression in chondrocytes requires $\mathrm{p} 38$, c-Jun $\mathrm{N}$-terminal kinase, and nuclear factor kappaB: differential regulation of collagenase 1 and collagenase 3 . Arthritis Rheum. 43:801-811.

21. Schorpp, M., et al. 1995. Structural organization and chromosomal localization of the mouse collagenase type I gene. Biochem. J. 308:211-217. 